\title{
VARIACIÓN EN CARACTERÍSTICAS DE INTÉRES AGRONÓMICO DENTRO DE UNA POBLACIÓN NATIVA DE TOMATE (Solanum lycopersicum L.)
}

\author{
VARIATION IN CHARACTERISTICS OF AGRICULTURAL INTEREST WITHIN A \\ NATIVE TOMATO (Solanum lycopersicum L.) POPULATION
}

\author{
Felipe Sanjuan-Lara ${ }^{1 *}$, Porfirio Ramírez-Vallejo² ${ }^{\dagger}$, Prometeo Sánchez-García $^{2}$, Manuel Livera-Muñoz $^{2}$, \\ Manuel Sandoval-Villa ${ }^{2}$, José C. Carrillo-Rodríguez ${ }^{3}$ y Catarino Perales-Segovia ${ }^{4}$
}

\begin{abstract}
${ }^{1}$ Centro de Bachillerato Tecnológico. Agropecuario. No. 79. Km 3.5 Camino a San Luis del Pino. 75960, Zinacatepec, Puebla. ${ }^{2}$ Colegio de Postgraduados, Campus

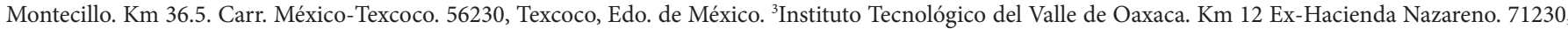
Santa Cruz Xoxocotlán, Oaxaca. ${ }^{4}$ Instituto Tecnológico El Llano. Km 18 Carr. Aguascalientes-San Luis Potosí. 20157, Aguascalientes, Aguascalientes.
\end{abstract}

*Autor para correspondencia (felisl15@hotmail.com)

\section{RESUMEN}

México es un país con gran diversificación de poblaciones silvestres de tomate (Solanum lycopersicum L.), pero poco estudiadas, por lo que se requiere explorar y conocer su potencial genético como fuente para mejorar la productividad, tolerancia a sequía, salinidad y resolver problemas fitosanitarios. Con el objetivo de determinar la variabilidad genética en 120 familias de tomate derivadas de una población nativa del Estado de Puebla, con base en características de interés agronómico, se sembraron y caracterizaron en un invernadero cubierto de malla antiáfidos a los $60 \mathrm{~d}$ después del trasplante. Los análisis de varianza detectaron diferencias $(P \leq 0.05)$ en las seis variables evaluadas, $y$ el análisis de componentes principales mostró que dos variables (calificación general y diámetro de tallo) describieron $59.7 \%$ de la variabilidad morfológica. La representación gráfica de los dos principales componentes permitió identificar a seis grupos de familias, distribuidas en los cuatro cuadrantes. Los Grupos I y VI concentraron a las familias sobresalientes en diámetro de tallo, altura de planta, número de racimos con fruto, con plantas sanas y uniformes. El Grupo II aglutinó 84 \% del total de las familias buenas; y los Grupos III y IV se caracterizaron por integrar familias de regulares a malas.

Palabras clave: Solanum lycopersicum, componentes principales, población nativa.

\section{SUMMARY}

México is a country with a great diversity of wild tomato (Solanum lycopersicum L.) populations. They have been, however, little studied and so it is necessary to explore and learn their genetic potential as a germ plasm source for improving productivity, tolerance to drought and salinity, and plant health problems. With the aim of determining genetic variability in 120 tomato families derived from 'Chino', a native population in the state of Puebla, and based on characteristics of agronomic interest, they were planted under greenhouse conditions and characterized $60 \mathrm{~d}$ after transplant. The greenhouse was covered with an aphid resistant mesh. Analyses of variance detected differences $(P \leq 0.05)$ in all six variables measured. The main component analysis showed that two variables (general grading and stem diameter) described 59.7 $\%$ of the morphological variability. The graphic representation of the two main components allowed us to identify six family groups, distributed in all four quadrants. Groups I and VI concentrated the families outstanding in stem diameter, plant height, number of fruit clusters, and healthy and uniform plants. Group II included $84 \%$ of the total good families; and Groups II and IV integrated families ranging from regular to bad.

Index words: Solanum lycopersicum, main components, native population.

\section{INTRODUCCIÓN}

La mayor diversidad de especies vegetales comestibles se concentra en países en desarrollo (Bastias, 2008). Una de estas especies es el tomate (Solanum lycopersicum L.), hortaliza de importancia económica domesticada en México, donde existe una amplia variabilidad morfológica, además de evidencias lingüísticas, arqueológicas y etnobotánicas (Rick y Fobes, 1975; Rick, 1979). Actualmente esta especie presenta una gran diversificación de poblaciones nativas, aunque éstas han sido escasamente documentadas en sus características físicas (Peralta y Spooner, 2007; Méndez et al., 2011); tampoco hay reportes sobre su potencial genético y aprovechamiento directo o como fuente de genes para el mejoramiento (Carrillo y Chávez, 2010).

En la actualidad es posible encontrar poblaciones nativas cultivadas en diferentes regiones agrícolas del país así como poblaciones nativas no cultivadas; estas últimas están ampliamente distribuidas en reservas ecológicas en las que se encuentran asociadas con cultivos, tales como el maíztomatillo (Zea mays-Physalis ixocarpa), en los cuales eventualmente se convierten en malezas (Rodríguez et al., 2009; Chávez et al., 2011). Esta distribución ha permitido a las poblaciones de tomate desarrollar características especiales de adaptación a factores bióticos y abióticos, que determinan la extensión de la variabilidad biológica de la especie (Ramanatha y Hodgkin, 2002; Álvarez et al., 2009).

Con relación a la variación morfológica de poblaciones nativas, estudios realizados en condiciones de invernadero con 49 poblaciones nativas de Oaxaca mostraron 
diferencias significativas en 19 características fenológicas y morfológicas de los órganos aéreos de la planta (Carrillo y Chávez, 2010). En contraste, al evaluarlas en campo, poblaciones silvestres de Michoacán no mostraron diferencias en color del fruto (rojo), color de la flor (amarillo), margen de los foliolos (aserrados) y hábito de crecimiento (rastrero-trepador) (Álvarez et al., 2009).

La acumulación de materia seca es una característica de importancia agronómica, ya que un incremento de biomasa con frecuencia se asocia con un incremento en el rendimiento, y las diferencias de biomasa entre genotipos se manifiestan aún en estado temprano de plántula (Utria et al., 2005). En diez líneas de tomate obtenidas de una población nativa, expuestas a tres concentraciones de solución nutritiva, se encontraron diferencias estadísticas en rendimiento, con niveles similares a los testigos de los tipos "bola" y "saladette" (González et al., 2012).

En genotipos de tomates nativos (Lycopersicon esculentum var. cerasiforme) del Estado de Guerrero evaluados en condiciones de invernadero con hidroponía, la mayoría fueron superiores al híbrido comercial en características de calidad de frutos (Juárez-López et al., 2009). En este sentido, la variabilidad genética se presenta en mayor grado en poblaciones nativas, como también reportaron RíosOsorio et al. (2014) en su estudio sobre el sistema de producción tradicional en la región de Tehuantepec-Juchitan, Oaxaca. Estos últimos autores indicaron que la variabilidad presente en morfotipos de frutos nativos y su producción se destina a los mercados regionales, lo que promueve la preservación in situ de los acervos genéticos locales de tomate.

A nivel mundial se estima que $80 \%$ de las muestras de germoplasma nativo de diferentes especies [calabaza $(\mathrm{Cu}$ curbita spp), maíz, chile (Capsicum annuum L.), entre otros] carecen de datos de caracterización y no han sido evaluadas agronómicamente. Esto significa que no existe información de las características morfológicas y fisiológicas de los recursos filogenéticos, lo que convierte a las colecciones en simples depósitos de material biológico sin mayor utilidad aparente (Bastias, 2008).

Para contribuir al conocimiento acerca del grado de variación intraespecífica de una población nativa de hermanos filiales de tomate, el presente trabajo se desarrolló con el objetivo de evaluar el grado de variación intrapoblacional en características de interés agronómico, en una muestra de 120 familias individuales derivadas de 'Chino', una población nativa de tomate del Estado de Puebla.

\section{MATERIALES Y MÉTODOS}

\section{Material vegetal y evaluación agromorfológica}

Se evaluaron 120 familias de tomate obtenidas por selección de plantas individuales sobresalientes en vigor de la planta y rendimiento de frutos en una población nativa conocida regionalmente como 'Chino'. La selección se realizó en un invernadero cubierto de malla antiáfidos denominado "casa sombra" en el municipio de Zinacatepec, Puebla, cuyas coordenadas geográficas son $18^{\circ} 17^{\prime} 30^{\prime \prime}$ y $18^{\circ} 23^{\prime} 00^{\prime \prime}$ N y $97^{\circ} 09^{\prime} 18^{\prime \prime}$ y $97^{\circ} 15^{\prime} 54^{\prime \prime} \mathrm{O}$, a una altitud de $1100 \mathrm{~m}$. Las familias se evaluaron en un invernadero provisto con sistema de riego por goteo, camas de suelo y acolchado plástico. Se sembraron dos semillas por cavidad en el mes de febrero de 2010 en charolas de unicel de 200 cavidades, con turba ("peat moss") como sustrato.

A los $25 \mathrm{~d}$ después de la siembra se trasplantaron 20 plántulas de cada familia a lo largo del surco. El riego se aplicó diariamente con una solución Steiner (1984), modificada de acuerdo con los resultados del análisis químico de agua y calculada en meq $\mathrm{L}^{-1}$ con las siguientes concentraciones de aniones y cationes: $12 \mathrm{NO}_{3}^{-}, 1 \mathrm{H}_{2} \mathrm{PO}_{4}^{-}, 0.5 \mathrm{SO}_{4}^{-2}, 6 \mathrm{~K}^{+}, 3$ $\mathrm{Ca}^{+2}$, y $1 \mathrm{Mg}^{+2}$.

La toma de las características de interés agronómico se hizo $60 \mathrm{~d}$ después del trasplante, con base en el descriptor para Solanum spp. del Instituto Internacional de Recursos Genéticos Vegetales (IPGRI, 1996): diámetro de tallo en la base $(\mathrm{cm})$, altura de planta $(\mathrm{cm})$, número de racimos con fruto, severidad de enfermedades, uniformidad y calificación agronómica general (Cuadros 1, 2, 3 y 4).

\section{Análisis estadístico}

Los tratamientos se distribuyeron en un diseño completamente al azar con cuatro repeticiones con 20 plantas cada una. Con los promedios de cada variable se efectuó un análisis de componentes principales y con este último se determinaron las variables de mayor valor descriptivo de la variación fenotípica, como propusieron Carrillo y Chávez (2010).

\section{RESULTADOS Y DISCUSIÓN}

Los análisis de varianza mostraron diferencias significativas $(P \leq 0.05)$ entre las familias en todas las variables. Los mayores coeficientes de variación se obtuvieron en número de racimos con fruto $20 \%$, severidad general $27 \%$, uniformidad $24 \%$, y calificación fenotípica general 30 \% (Cuadro $5)$, valores que indican una amplia variabilidad fenotípica dentro de la población. Resultados similares se obtuvieron en diferentes poblaciones de tomate evaluadas en condiciones de invernadero en el Estado de Oaxaca, que mostraron 
Cuadro 1. Características de interés agronómico evaluadas en una población de 120 familias provenientes de 'Chino', una población nativa de tomate del Estado de Puebla, 2010, (IPGRI, 1996).

\begin{tabular}{lll}
\hline Características & Variables & Unidad \\
\hline Morfología (planta) & Diámetro de tallo & $\mathrm{cm}$ \\
Longitud & Longitud de planta $(60 \mathrm{ddt})$ & $\mathrm{cm}$ \\
Componente de rendimiento & Núm. de racimos con fruto & \\
\hline
\end{tabular}

Cuadro 2. Escala de reacción utilizada en la calificación fitopatológica (severidad general) de 120 familias provenientes de 'Chino', una población nativa de tomate del Estado de Puebla, 2010.

\begin{tabular}{|c|c|c|c|}
\hline Calificación & Severidad (\%) & Categoría & Descripción \\
\hline 9 & 0 & \multirow{3}{*}{ Sin severidad } & \multirow{3}{*}{ Síntomas no visibles o muy leves. } \\
\hline 8 & 10 & & \\
\hline 7 & 20 & & \\
\hline 6 & 30 & \multirow{3}{*}{ Severidad intermedia } & \multirow{3}{*}{$\begin{array}{l}\text { Síntomas visibles o conspicuos que solo } \\
\text { ocasionan un daño limitado. }\end{array}$} \\
\hline 5 & 40 & & \\
\hline 4 & 50 & & \\
\hline 3 & 60 & \multirow{4}{*}{ Severidad alta } & \multirow{4}{*}{$\begin{array}{l}\text { Síntomas severos a muy severos que ocasio- } \\
\text { nan pérdidas de rendimiento considerables } \\
\text { o la muerte de la planta. }\end{array}$} \\
\hline 2 & 70 & & \\
\hline 1 & 80 & & \\
\hline 0 & $90-100$ & & \\
\hline
\end{tabular}

Cuadro 3. Escala de calificación para uniformidad general de 120 familias provenientes de 'Chino', una población nativa de tomate del Estado de Puebla, 2010.

\begin{tabular}{lcc}
\hline Calificación & Categoría & Descripción \\
\hline $0-30$ & Uniforme & Plantas uniformes en longitud, floración, amarre de fruto y vigorosidad. \\
$31-60$ & $\begin{array}{c}\text { Uniformidad } \\
\text { intermedia }\end{array}$ & $\begin{array}{c}\text { Algunas plantas de la familia presentan variación de longitud de planta, floración, } \\
\text { amarre de fruto y poco vigorosas. }\end{array}$ \\
61-100 & Heterogeneidad & $\begin{array}{c}\text { Más de la mitad de la familia presenta variación en longitud de planta, floración, } \\
\text { amarre de fruto y sin vigor. }\end{array}$ \\
\hline
\end{tabular}

Cuadro 4. Escala de calificación general de 120 familias provenientes de 'Chino', una población nativa de tomate del Estado de Puebla, 2010.

\begin{tabular}{lcr}
\hline Calificación & Categoría & Descripción \\
\hline $1-2$ & Buena & $\begin{array}{r}\text { Plantas sin severidad, uniformes en longitud, buen amarre de fruto y vigorosas. } \\
\text { Plantas con síntomas visibles pero daño limitado, variación en longitud, raci- } \\
\text { mos con poco amarre de fruto y poco vigorosas. }\end{array}$ \\
Regular & $\begin{array}{r}\text { Plantas con severidad que ocasionan perdida de rendimiento, más de la mitad } \\
\text { presentan variación en longitud, amarre de fruto y sin vigor. }\end{array}$ \\
\hline
\end{tabular}




\begin{tabular}{|c|c|c|c|c|}
\hline Variables & Promedio & ${ }^{\dagger} \mathrm{DE}$ & CV (\%) & Intervalos \\
\hline Diámetro de tallo & 8.19 & 0.76 & 9 & 7 a 11 \\
\hline Altura de planta & 96.74 & 8.10 & 8 & 70 a 116 \\
\hline Número de racimos con fruto & 1.91 & 0.37 & 20 & 1 a 3 \\
\hline Severidad general & 17.45 & 4.71 & 27 & 10 a 35 \\
\hline Uniformidad & 17.79 & 4.19 & 23 & 10 a 30 \\
\hline Calificación general & 1.85 & 0.56 & 30 & 1 a 3 \\
\hline
\end{tabular}

Significativamente diferente $(\mathrm{P} \leq 0.05)$. ${ }^{\dagger}$ Desviación estándar.

coeficientes de variación de $26 \%$ en número de frutos por racimo, $12 \%$ en diámetro de tallo, y $8 \%$ en altura de planta (Carrillo y Chávez, 2010; Chávez et al., 2011).

La mayor parte de la diversidad y variabilidad de los tomates cultivados se encuentra también en tomates nativos, tanto en calidad de frutos como en características morfo agronómicas de la planta, como lo demostraron Restrepo et al. (2006) al encontrar coeficientes de variación entre 23 y $52 \%$ en días a inicio de floración, altura de planta al primer racimo, altura de planta en duodécima flor, altura total, diámetro de tallo y frutos por inflorescencia, en accesiones silvestres de Lycopersicon spp. Por su parte, en 30 accesiones de tomate nativo tipo "cereza", Boada et al. (2010) encontraron diferencias significativas en sólidos solubles, con valores extremos de 3.8 a 6.3 , y rendimientos de $60 \mathrm{t} \mathrm{ha}^{-1}$.

En el análisis de componentes principales, los dos primeros componentes (calificación general y diámetro de tallo) fueron las de mayor valor descriptivo al explicar $59.7 \%$ de la variación fenotípica total en las familias de este tomate nativo (Cuadro 6). Las diferencias entre las familias evaluadas estuvieron definidas por características morfológicas y tolerancia a enfermedades, asociadas con vigor del tallo y uniformidad en crecimiento. Restrepo et al. (2006) encontraron en los primeros dos componentes (días a inicio de floración y diámetro de tallo) la explicación de $76.3 \%$ de la variación total, en germoplasma silvestre de (Lycopersicon spp). Durante el proceso de evolución y diversificación, los tomates silvestres han desarrollado múltiples características que les permiten sobrevivir en condiciones extremas y tolerar plagas y enfermedades (Eigenbrode et al., 1993; Nuez et al., 1995).

Las familias de tomate provenientes del Estado de Puebla llamadas localmente "chino" se dispersaron en los cuatro cuadrantes como resultado de la amplia variabilidad intrapoblacional existente en la población original, y formaron seis grupos con base en los dos primeros componentes principales (calificación general y diámetro de tallo) (Figura 1). El Grupo I estuvo constituido por las familias 33 y 47 que se ubicaron en los cuadrantes I y IV. La familia 33 presentó mayores valores de diámetro de tallo y altura de planta, y es considerada como de buen fenotipo; y aunque la 47 presentó buen diámetro de tallo y altura de planta, se caracterizó como de fenotipo regular.

El Grupo II se conformó con $84.16 \%$ del total de las familias, que se dispersaron en los cuadrantes I, II, III y IV. En los cuadrantes I y IV las familias tuvieron en promedio 2.25 racimos con fruto, mientras que en los cuadrantes II y III el promedio fue de 1.75 racimos con fruto; en ambos cuadrantes la calificación general fue de 1.5 a 2, consideradas como plantas de buen fenotipo. En el Grupo III se ubicaron 9 familias (19, 58, 117, 54, 18, 60, 7, 9 y 62) dispersas en los cuadrantes I y II, cuyo promedio de racimos con fruto estuvo entre 1.75 y 2 , con una calificación general de 3 y 3.5 , que las califica fenotípicamente como de regulares a malas.

El Grupo IV se ubicó en el cuadrante II, cuya cantidad de racimos con fruto está por debajo de la media (1.91), y se clasificaron como familias sin síntomas severos de enfermedades, con plantas pequeñas y poco vigorosas cuya calificación general fue de 3 , clasificadas como plantas de fenotipo regular. En el Grupo V se ubicaron las familias 97 y 106 con el mayor número de racimos y frutos $(2.25$ y 3$)$, con calificación general de 1 (plantas sanas, uniformes en altura de planta, buen amarre de fruto), aunque poco vigorosas en diámetro de tallo.

En el Grupo VI, dos familias (98 y 105) se ubicaron en el cuarto cuadrante, que resultaron sobresalientes por tener mayores valores de diámetro de tallo, altura de planta, número de racimos con fruto, sanidad y uniformidad.

Es importante mencionar que el uso de germoplasma silvestre se inició en Estados Unidos de América desde 1930 (Rick, 1986), mientras que en México poco se hace al 
Cuadro 6. Vectores y valores propios del análisis de componentes principales (CP) con las variables de mayor valor descriptivo de la varianza total de 120 familias provenientes de 'Chino', una población nativa de tomate del Estado de Puebla, 2010.

\begin{tabular}{lcccc}
\hline Variables agromorfológicas & CP1 & CP2 & CP3 & CP4 \\
\hline Calificación general & 0.573 & 0.157 & 0.159 & -0.115 \\
Diámetro de tallo & -0.152 & 0.704 & 0.248 & -0.609 \\
Severidad general & 0.503 & 0.297 & 0.134 & 0.255 \\
Uniformidad general & 0.501 & 0.076 & -0.009 & 0.220 \\
Altura de planta & -0.371 & 0.367 & 0.466 & 0.674 \\
Número de racimos con fruto & -0.068 & 0.499 & -0.822 & 0.215 \\
Valores propios & 2.310 & 1.273 & 0.938 & 0.632 \\
Varianza explicada (\%) & 0.385 & 0.212 & 0.156 & 0.105 \\
Varianza acumulada (\%) & 0.385 & 0.597 & 0.753 & 0.858 \\
\hline
\end{tabular}

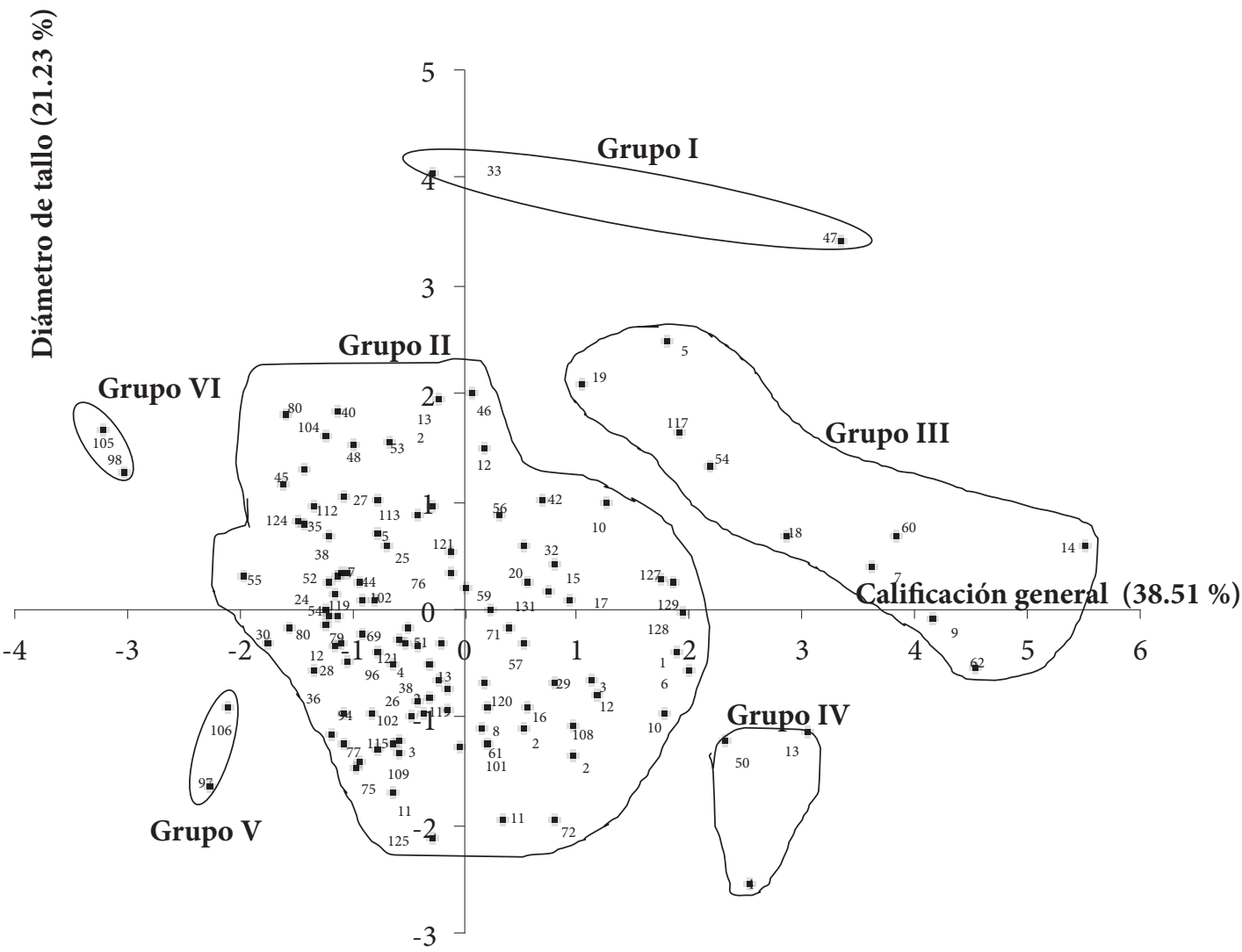

Figura 1. Dispersión de 120 familias de tomate nativo “Chino” del Estado de Puebla con base en los dos primeros componentes. 
respecto a pesar de ser centro de domesticación y diversificación. Antes de promover su utilización en el mejoramiento del tomate, es necesario conocer la variabilidad genética que se conserva in situ. No hay reportes específicos de caracterización morfológica del tomate semi-domesticado y silvestre en México, y sólo se han reportado 20 accesiones en el banco de germoplasma de la Universidad Autónoma Chapingo (Molina y Córdoba, 2006).

\section{CONCLUSIONES}

Las 120 familias provenientes de una población de tomate nativo del Estado de Puebla presentaron mayor variación intrapoblacional, sobre todo en número de racimos con fruto $(19.6 \%)$, severidad general $(27.0 \%)$, uniformidad (23.5 \%) y calificación general (30.3\%). Los dos primeros componentes del análisis de componentes principales (calificación general y diámetro de tallo) explicaron 59.7 \% de la variación fenotípica.

\section{BIBLIOGRAFÍA}

Álvarez H. J. C., M. Cortez y R. García (2009) Exploración y caracterización de poblaciones silvestres de jitomate (Solanaceae) en tres regiones de Michoacán, México. Polibotánica 28:139-159.

Bastias M. E. (2008) Biodiversidad y recursos filogenéticos en la agricultura. IDESIA 26:5-7.

Boada H. M. Y., J. L. Mejía, N. Ceballos y F. Javier (2010) Evaluación agronómica de treinta introducciones de tomate silvestre tipo cereza (Solanun lycopersicum L.). Agronomía 18:59-67.

Carrillo R. J. C. y J. L. Chávez (2010) Caracterización agromorfológica de muestras de tomate de Oaxaca. Revista Fitotecnia Mexicana 33:1-6.

Chávez S. J. L., J. C. Carrillo, A. M. Vera, E. Rodríguez y R. Lobato (2011) Utilización Actual y Potencial del Jitomate Silvestre Mexicano. Subsistema Nacional de Recursos Fitogéneticos para la Alimentación y la Agricultura (SINAREFI), Secretaría de Agricultura, Ganadería, Desarrollo Rural, Pesca y Alimentación, CIIDIR-Unidad Oaxaca del Instituto Politécnico Nacional e Instituto Tecnológico del Valle de Oaxaca, México. 72 p.

Eigenbrode S. D., J. T. Trumble and R. A. Jones (1993) Resistence to beet armyworm (Spodopteraexigua [Hubner]), hemipterans, and $L i$ riomyza spp. In Lycopersicum. Journal of the American Society for Horticultural Science 118:525-530.

González F., M. Sandoval, P. Sánchez, P. Ramírez and M. N. Rodríguez (2012) Yield of native genotypes of tomato as affected by electrical conductivity of nutrient solution. Acta Horticulture 947:69-76.

IPGRI, International Plant Genetic Resources Institute (1996) Descriptors for Tomato. FAO/Bioversity Multi-Crop Passport Descrip- tors V.2 [MCPD V.2]. 44 p. Available at: indoplasma.or.id/descriptor/IPGRI/descriptor tomat (Enero 2014).

Juárez-López P., R. Castro-Brindis, T. Colinas-León, P. Ramírez-Vallejo, M. Sandoval-Villa, D. W. Reed, L. Cisneros-Zevallos y S. King (2009) Evaluación de calidad de frutos de siete genotipos nativos de jitomate (Lycopersicon esculentum var. cerasiforme). Revista Chapingo Serie Horticultura 15:5-9.

Méndez I. I., M. Areli, G. Vera, J. L. Chávez y J. C. Carrillo R. (2011) Calidad de frutos en variedades nativas Mexicanas de tomate (Lycopersicon esculentum Mill). Revista de la Facultad de Química Farmacéutica 18:26-32.

Molina M. J. C. y L. Córdova T. (2006) Conservación ex situ. In: Recursos Fitogenéticos de México para la Alimentación y la Agricultura. Informe Nacional 2006. J. C. Molina M., L. Córdova T. (eds). Secretaría de Agricultura, Ganadería, Desarrollo Rural, Pesca y Alimentación y Sociedad Mexicana de Fitogenética, A.C. Chapingo, México. pp:59-100

Nuez V., A. Rodríguez, J. Tello, J. Cuartero y B. Segura (1995) El cultivo del Tomate. Ed. Mundi-Prensa. Bilbao, España. 793 p.

Peralta I. E. and D. M. Spooner (2007) History, origin and early cultivation of tomato (Solanaceae). In: Genetic Improvement of Solanaceous Crop, Vol. 2: Tomato. M. K. Razdan, A. K. Razdan and A. K. Mattoo (eds). Science Publishers. Enfield. New Hampshire, USA. pp:1-24.

Ramanatha R. V. and T. Hodgkin (2002) Genetic diversity and conservation and utilization of plant genetic resources. Plant Cell and Tissue Organ Culture 68:1-19.

Restrepo S., E. Fernando, C. Vallejo, F. Alirio y M. Lobo (2006) Evaluación de la resistencia al pasador del fruto Neoleucinodes eleganlis y caracterización morfoagronómica de germoplasma silvestre de Lycopersicon spp. Acta Agronómica 55:1-10.

Rick C. M. (1979) Biosystematic studies in Lycopersicon and closely related species of Solanum. In: The Biology and Taxonomy of the Solanaceae. J. Hawahkes, G. Lester A. D. Skeldinng (eds). Linnean Society of London. London, U. K. pp:667-677.

Rick C. M. (1986) Germplasm resources in the wild tomato species. Acta Horticulturae 190:39-48.

Rick C. M. and J. Fobes (1975) Allozyme variation in cultivated tomato and closely related species. Bulletin of the Torrey Botanical Club 102:376-384.

Ríos-Osorio O., J. L. Chávez-Servia y J. C. Carrillo-Rodríguez (2014) Producción tradicional y diversidad de tomate (Solanum lycopersicum L.) Nativo: un estudio de caso en Tehuantepec-Juchitán, México. Agricultura, Sociedad y Desarrollo 11: 35-51.

Rodríguez G. E., D. Vargas, J. J. Sánchez, R. Lépiz, A. Rodríguez, J. A. Ruíz, P. Puente y R. Miranda (2009) Etnobotánica de Sola num lycopersicum var. ceraciforme en el occidente de México. Naturaleza y Desarrollo 7:45-57.

Steiner A. A. (1984) The Universal Nutrient Solution. In: Proc. 6th International Congress Soilless Culture. pp:633-649.

Utria E. R. I., A. Cabrera, D. Morales y A. Lores (2005) Crecimiento de plántulas de tomate (Lycopersicon esculentum Mill.) cultivadas en diferentes sustratos y niveles de abastecimiento hídrico. $\mathrm{Cul}$ tivos Tropicales 26:31-38.

Villa D., W. Reed, L. Cisneros-Zevallos y S. King (2009) Evaluación de calidad de frutos de siete genotipos nativos de jitomate ( $\mathrm{Lyco}$ persicon esculentum var. cerasiforme). Revista Chapingo Serie Horticultura 15:5-9. 